\title{
Myelodysplastic Syndrome with Ring Sideroblasts
}

National Cancer Institute

\section{Source}

National Cancer Institute. Myelodysplastic Syndrome with Ring Sideroblasts. NCI

Thesaurus. Code C4036.

A myelodysplastic syndrome characterized by an anemia in which $15 \%$ or more of the erythroid precursors are ring sideroblasts. The ring sideroblast is an erythroid precursor in which one third or more of the nucleus is encircled by granules which are positive for iron stain. (WHO, 2001) 